\title{
The expression of high-mobility group protein box 1 correlates with the progression of non-small cell lung cancer
}

\author{
XIAOKUN SHEN ${ }^{1}$, LINGZHI HONG $^{1}$, HUIMING SUN ${ }^{1}$, MINKE SHI $^{2}$ and YONG SONG ${ }^{1}$ \\ ${ }^{1}$ Department of Respiratory Medicine, Jinling Hospital, Nanjing University School of Medicine, \\ Nanjing 210002; ${ }^{2}$ Department of Cardiothoracic Surgery, Affiliated Drum Tower Hospital, \\ Nanjing University School of Medicine, Nanjing 210008, P.R. China
}

Received February 25, 2009; Accepted April 13, 2009

DOI: $10.3892 /$ or_00000468

\begin{abstract}
High-mobility group protein box 1 (HMGB1) released from dying tumor cells promotes tumor progression, on the other hand HMGB1 activates dendritic cells and triggers anti-neoplastic response of T-cells in chemotherapy and radiotherapy. HMGB 1 expression is up-regulated in many kinds of tumors. To investigate HMGB1 expression in non-small cell lung cancer, 63 patients were enrolled and tumor tissues were collected with matched normal lung tissues. HMGB1 mRNA and protein levels were quantified with real-time PCR, Western blots and immunohistochemistry. The HMGB 1 expression in tumor tissue was lower than in matched normal lung tissue at mRNA levels (Paired t-test, $\mathrm{t}<0.001$ ) and the down-regulation in stage III-IV patients was also significantly greater than one in stage I-II (one-way ANOVA, p=0.005). At protein level, HMGB1 expression was also down-regulated. Low HMGB 1 expression in non-small cell lung cancer may explain the poor therapeutical outcome during chemotherapy and radiotherapy.
\end{abstract}

\section{Introduction}

High-mobility group protein box 1 (HMGB1) is a member of the high-mobility group protein superfamily with a $27 \mathrm{kDa}$ molecular mass. As a non-histone chromatin protein, HMGB1 plays an important role in DNA architecture and

Correspondence to: Dr Yong Song, Department of Respiratory Medicine, Jinling Hospital, Nanjing University School of Medicine, Nanjing 210002, P.R. China

E-mail: yong_song6310@yahoo.com

Abbreviations: HMGB1, high-mobility group protein box 1; AGER, advanced glycosylation end product-specific receptor; TLR, Toll-like receptor; DCs, dendritic cells; NSCLC, non-small cell lung cancer; GAPDH, glyceraldehyde-3-phosphate dehydrogenase

Key words: high-mobility group protein box 1, non-small cell lung cancer, progression, real-time PCR transcriptional regulation (1). When released actively by immune cells or passively by necrotic cells to extracellular environment, HMGB1 functions to recruit inflammatory cells and to mediate signals between natural killer cells, dendritic cells and macrophages by interacting with AGER (advanced glycosylation end product-specific receptor) and/or TLR2/4 (Toll-like receptor) to activate the NF-кB pathway. As a damage-associated molecular pattern molecule (DAMP), HMGB1 promotes inflammation, stimulates tissue repair and is involved in many pathological conditions, such as sepsis, arthritis, and ischemia-reperfusion injury (2-4).

HMGB1 was found to be related to tumor development from the discovery of its action in the modulation of neurite outgrowth many years ago (5) and now more and more studies show that HMGB1-AGER interactions mediate cancer cell's proliferation, invasion and migration $(6,7)$. Mitola et al demonstrated that HMGB1 could stimulate tumor angiogenesis in vitro and in vivo (8). As a so-called danger-signaling cytokine, HMGB 1 promotes tumor progression. Upregulated expression of HMGB1 has been found in many tumor types, including breast cancer $(9,10)$, hepatocellular carcinoma (11), melanoma $(12,13)$, gastric cancer $(14)$, nonHodgkin lymphoma (15) and colorectal cancer (16).

However, a previous study revealed that the relationship between HMGB1 and tumor had an additional totally opposite aspect. Apetoh et al revealed that during chemotherapy and radiotherapy, dying cancer cells secreted HMGB1, that acted on the Toll-like receptor 4 (TLR4) expressed on the dendritic cells and efficiently processed and crosspresented the antigen through TLR4 and its adaptor MyD88 from dying tumor cells to activate the tumor antigen-specific T-cell immunity that was essential for therapy (17). They found that breast cancer patients who carried a loss-offunction TLR4 allele, the key figure of the immune response, relapsed more quickly after radiotherapy and chemotherapy than those carrying the normal TLR4 allele. They also found that the depletion of HMGB1 from doxorubicin- or oxaliplatintreated tumor cells using neutralizing antibodies or HMGB1specific siRNAs compromised the efficacy of anti-tumor vaccination. Therefore HMGB1 has a paradoxical dual effect: on one hand it promotes tumor progress, on the other hand it triggers protective anti-cancer responses of T-cells.

Up-regulated HMGB1 expression is found in almost all type of tumors detected together with advanced glycosylation 
end product-specific receptor (AGER) expression, supporting the idea that HMGB 1 is a danger-signaling cytokine. Interestingly, lung cancer is the exception with reduced expression of AGER (18). This prompted us to investigate the HMGB1 expression in lung cancer. HMGB1 expression was quantified in non-small cell lung cancer. To our surprise, as a whole, HMGB1 expression was down-regulated and the down-regulation was related with tumor stage.

\section{Materials and methods}

Patients and tissue specimens. Tumor tissues (TT) and matched tumor-free tissues (TF) $>5 \mathrm{~cm}$ far from the tumor edge were obtained from 63 Chinese lung cancer patients with surgical therapy in the Department of thoracic Surgery of Jinling Hospital from 2006 to 2008. All the samples were collected immediately after surgical resection. Necrotic or hemorrhagic tissues were excluded. Normal lung tissues from 4 donors (a gift from $\mathrm{Dr} \mathrm{Yu}$ Decai) and 9 patients with no evidence of lung cancer served as healthy controls (HC). The Research Ethics Committee of Jinling Hospital approved this protocol and verbal consent was obtained from all participants. Lung cancer is classified by histological type, grade and stage according to WHO standards. Tissues were snap-frozen and kept in liquid nitrogen until use (protein and RNA isolation), or were fixed in $10 \%$ formalin and embedded in paraffin for immunohistochemical study.

RNA extraction and cDNA synthesis. Total RNA was isolated from frozen tissue with TRIzol (Invitrogen). With random hexamer primers, $2 \mu \mathrm{g}$ RNA was reverse transcribed to cNDA with PrimeScript ${ }^{\mathrm{TM}}$ first-strand cDNA synthesis kits (Takara).

Real-time PCR. Primers for HMGB1 and glyceraldehyde-3phosphate dehydrogenase (GAPDH) were designed and synthesized by Takara Inc. The basic information on the primers including gene name, NCBI reference, forward primer, reverse primer, product size (bp) was as follows, respectively: HMGB1, NM_002128, TGCAGATGACAAG CAGCCTTATG, TTTCAGCCTTGACAACTCCCTTT, 128; GAPDH, NM_002046, GCACCGTCAAGGCTGAGAAC, TGGTGAAGACGCCAGTGGA, 138. Real-time PCR was performed in triplicate for each sample in a $25-\mu 1$ reaction mixture, which consisted of template DNA $(2 \mu \mathrm{l})$ and primers $(0.2 \mu \mathrm{M})$, ROX Reference Dye II (1x), $\mathrm{dH}_{2} \mathrm{O}(9.0 \mu \mathrm{l})$ and SYBR $^{\circledR}$ Premix Ex Taq (1x, SYBR Premix Ex Taq Kit, Takara). PCR was performed on Stratagene Mx3005P instrument using the following thermal settings: one cycle of $10 \mathrm{sec}$ at $95^{\circ} \mathrm{C}$ and 45 cycles of $5 \mathrm{sec}$ at $95^{\circ} \mathrm{C}$ and $20 \mathrm{sec}$ at $60^{\circ} \mathrm{C}$. The relative expression ratio (RR) of HMGB1 gene was calculated based on the $\mathrm{Ct}$ comparative with a reference gene (GAPDH) in a sample.

Western blot analysis. For protein analysis, samples were homogenized in lysis buffer $(0.1 \%$ SDS, $50 \mathrm{mM}$ Tris- $\mathrm{HCl}$, $\mathrm{pH} 7.5,1 \% \mathrm{NP}-40,150 \mathrm{mM} \mathrm{NaCl}, 1 \mathrm{mM}$ Triton X-100, $1 \mathrm{mM}$ EDTA) containing complete protease inhibitor (PMSF+P8340). Protein concentration was measured by the BCA protein assay (Sigma). A total of $100 \mu \mathrm{g}$ protein was separated by $10-5 \%$ SDS-PAGE. Protein was then transferred to nitrocellulose membrane and these were saturated by incubating for $2 \mathrm{~h}$ with $5 \%$ non-fat dry milk in PBS $/ 0.1 \%$ Tween- 20 in $37^{\circ} \mathrm{C}$. The membranes were then incubated with the rabbit polyclonal anti-HMGB 1 antibody (Abcom) overnight at $4^{\circ} \mathrm{C}$. After three washes (5 min each) with PBS/0.1\% Tween-20, membranes were incubated with anti-rabbit immunoglobulin coupled to peroxidase $(\mathrm{Abcom})$ in $37^{\circ} \mathrm{C}$. After $1 \mathrm{~h}$ of incubation the membranes were washed four times (5 min each) with PBS/ $0.1 \%$ Tween-20 and the blots were developed using the chemiluminescence procedure (Amersham Bioscence). Polyclonal anti- $\beta$-actin antibody served as control.

Immunohistochemistry of paraffin-embedded sections. IHC staining was performed using standard protocols. Briefly, all paraffin sections, 4- $\mu \mathrm{m}$ in thickness, were baked for $1 \mathrm{~h}$ at $60^{\circ} \mathrm{C}$. Sections were deparaffinized with xylene and rehydrated with graded ethanol to distilled water. Sections were submerged in citrate buffer $(\mathrm{pH}$ 6.0) and subjected to microwave treatment, then treated with $3 \% \mathrm{H}_{2} \mathrm{O}_{2}$ for $15 \mathrm{~min}$ to block the endogenous peroxidase. Rabbit polyclonal antiHMGB1 antibody (Abcom) (1:100) was incubated with the sections for $1.5 \mathrm{~h}$ at $37^{\circ} \mathrm{C}$. After washing with PBS, the sections were incubated with intensifier for $20 \mathrm{~min}$ at room temperature. Secondary goat anti-rabbit polyclonal antibody was incubated with sections for $30 \mathrm{~min}$ at room temperature. For colour reactions, diaminobenzidine (DAB) was used. As negative controls, the antibody was replaced by normal rabbit serum.

Statistical analysis. Data were expressed as mean \pm standard deviation with the range given in parentheses. Statistical analysis was performed using the t-tests, ANOVA and paired t-test when data were normally distributed. All statistical procedures were performed using SPSS (Version 15.0 SPSS Inc, Chicago). Values of $\mathrm{p}<0.05$ were considered as statistically significant.

\section{Results}

Characteristics of the NSCLC patients. In 63 patients (median age $58 \mathrm{yrs}$ ) who underwent operative treatment (47 cases for lobectomy, 9 for pneumonectomy, 4 for segmental resection and 3 for sleeve lobectomy), the tumor size was $4.26 \pm 1.26 \mathrm{~cm}$ (range: 1-9 cm). Forty-nine patients underwent sequential therapy after surgery resections and 3 patients with N2 stage underwent induction chemotherapy before resections (the three patients degraded to N0-1 after the induction chemotherapy). Thirty-four patients (54\%) were smokers. Other characteristics are summarized in Table I.

Expression of HMGB1 in lung cancer tissues. The relative mRNA level of HMGB1 gene (HMGB1 per GAPDH, mean $\pm \mathrm{SD})$ is $1.09 \pm 0.53$ in $\mathrm{TT}, 2.24 \pm 1.14$ in $\mathrm{TF}$ and $2.15 \pm 1.76$ in $\mathrm{HC}$, respectively. HMGB1 in TT was significantly lower than in HC $(n=13)$ and TF (one-way ANOVA, $\mathrm{p}<0.001)$. Paired t-test showed that HMGB1 in TT was significantly lower than in TF ( $\mathrm{p}<0.001)$ (Fig. 1A). In summary, HMGB1 mRNA level is overall down-regulated in tumor samples of NSCLC patients.

In addition, 33 paired samples were analyzed by Western blots. In protein level, the expression of HMGB1 was also 
Table I. Characteristics of lung cancer patients in this study.

\begin{tabular}{lr}
\hline Gender & \\
Male & 51 \\
Female & 12 \\
Age & \\
$\geq 60$ & 28 \\
$<60$ & 35
\end{tabular}

Histological type

Adenocarcinoma

Squamous cell carcinoma

Adenosquamous carcinoma

BAC

Large cell

Carinoid

Differentiation

Well

Moderately

Poorly

Stage

I

II

III

IV
A

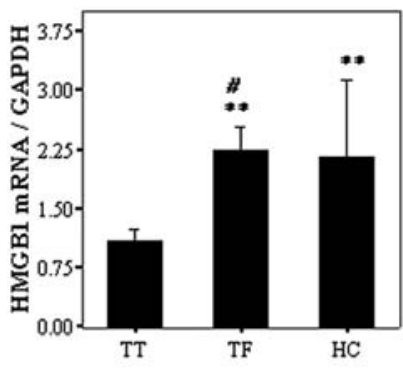

B

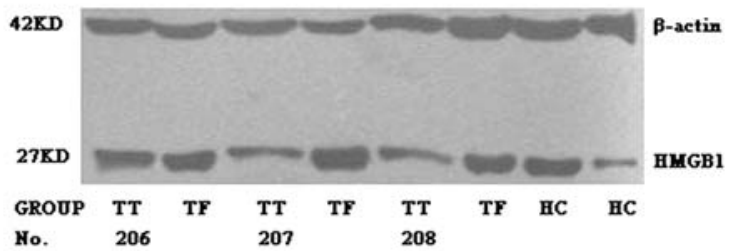

Figure 1. HMGB1 expression level in TT and TF. (A) Relative level of HMGB 1 mRNA in TT $(n=63)$, TF and HC $(n=13) .\left({ }^{* *} \mathrm{P}<0.001\right.$, vs. TT; ${ }^{\#} \mathrm{P}<0.001$, vs. TT). (B) Proteins in lanes 1-2 were extracted from TT and TF of patient 206, respectively; lanes 3-4 from TT and TF of patient 207; lanes 5-6 from TT and TF of patient 208; lanes 7-8 from normal tissues of two healthy controls. (C) Relative level of HMGB1 protein in TT $(n=33)$, TF and $\mathrm{HC}(\mathrm{n}=12) .{ }^{* * *} \mathrm{P}>0.05$, vs. TT; ${ }^{~} \mathrm{P}<0.05$, vs. TT) columns, mean; bars, SD.

A

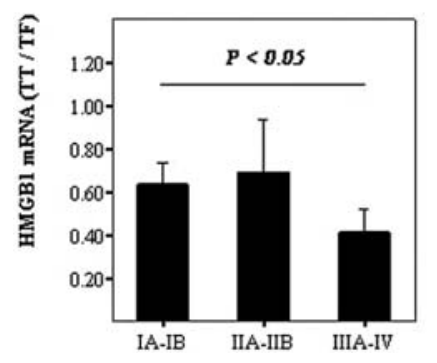

B

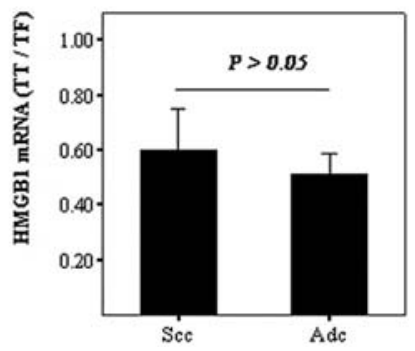

down-regulated in tumor tissues. The protein level of HMGB1 (HMGB1 per $\beta$-actin, mean \pm SD) is $0.64 \pm 0.43$ in TT, $0.90 \pm 0.54$ in TF and $0.91 \pm 0.55$ in $\mathrm{HC}$, respectively. Paired t-test showed that HMGB1 in TT was significantly lower than in TF $(\mathrm{p}=0.004)$. HMGB1 in TT was also lower than in $\mathrm{HC}(\mathrm{n}=13)$, but in one-way ANOVA, $\mathrm{p}=0.073$ (Fig. 1B and $\mathrm{C}$ ).

Down-regulation of the HMGB1 expression correlates with progression of NSCLC. The association between HMGB1 expression and clinical characteristics of patients was analyzed. There were no significant correlations between HMGB 1 expression and gender, age, pathological classifications ( $>0.05)$. However, III-IV stage patients show significantly less expression level of HMGB 1 than those in I-II stage $(\mathrm{p}=0.005)$ (Fig. 2). Of the patients in stage III, 3 patients with N2 stage who underwent induction chemotherapy before resections show no difference with others in HMGB 1 expression levels.

Distribution of HMGB1 in the lung tissues of NSCLC. Ten paired samples ( 3 pairs are adenocarcinoma and the other 7 pairs are squamous cell carcinoma) were analyzed in IHC. One adenocarcinoma sample had $30-50 \%$ positively stained, two squamous cell carcinoma samples had $<10 \%$ positively stained and seven tumor samples had $>50 \%$ tumor cells positively stained. All matched normal tissues were positively stained. In normal lung alveolar epithelial cells, the nucleolus was strongly stained and the cytoplasm stained moderately. However, in tumor tissues, only stained nucleolus could be found (Fig. 3).

Figure 2. The association between HMGB 1 expression and clinical characteristics of patients. (A) TNM stage-dependent expression of HMGB1 was evaluated from mRNA analyses of all NSCLC patients in relation to the HMGB1 level in the paired normal lung tissues. The result revealed a significant down-regulation of HMGB1 in NSCLC with increased TNM stage. (B) Pathological style-dependent expression of HMGB1. The result revealed no difference between the expression of HMGB1 in squamous cell carcinoma and in adenocarcinoma.

\section{Discussion}

We showed that HMGB1 expression was decreased in human non-small cell lung cancer tissues. At mRNA level, the HMGB1 expression decreased greatly and the differences between tumor tissue, matched normal tissue and healthy control were significant. At protein level, HMGB1 expression also decreased in tumor tissues in spite of several exceptions, but the difference was not significant. Some steroid hormones are linked to the transcriptional regulation of HMGB1 gene, including glucocorticoids, estrogens and progestins. In macrophages and monocytes, HMGB 1 transcription is regulated by IFN- $\gamma, \mathrm{TNF}-\alpha$ and TGF- $\beta$ (19). 


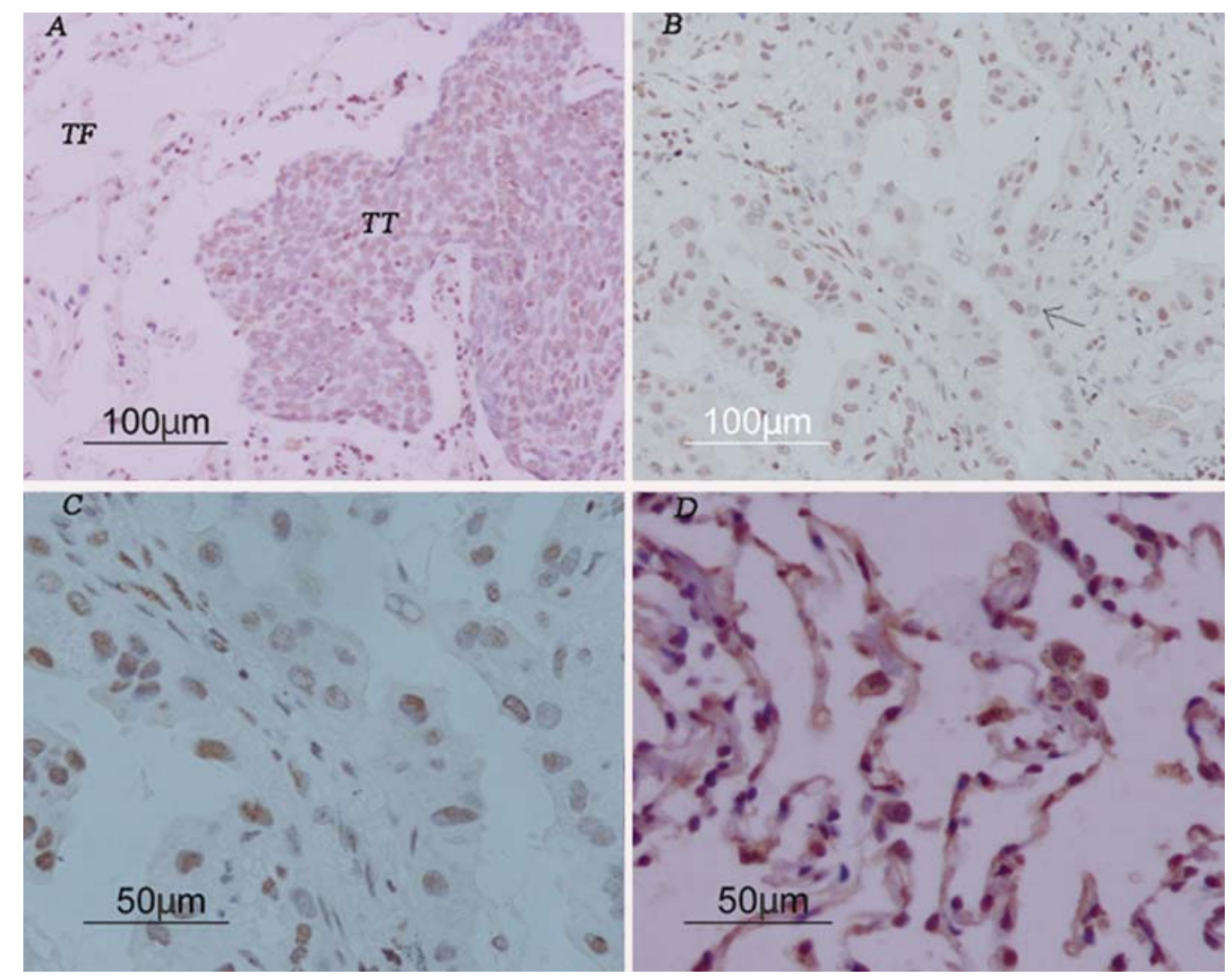

Figure 3. The expression and distribution of HMGB1 in NSCLC tissues with immunohistochemistry analyses. (A) HMGB1 expression in squamous cell carcinoma tissue and matched normal lung tissue. (B) HMGB1 expression was weak in some adenocarcinoma tumor cells (arrows). (C) Only the nucleoli were stained in positively-stained tumor cells. (D) The nucleoli and cytoplasm were both stained in normal lung tissue.

In cancer cells, down-regulation of some gene expression is commonly associated with the mutations or aberrant DNA methylation of the promoter. There are no previous studies on the promoter mutations of HMGB1, but one large $\mathrm{CpG}$ island (2000 bp) does exist in the beginning of HMGB1 gene including the promoter and enhancer. More studies need to be done to find out whether aberrant DNA methylation exists and reduces the expression of HMGB1 in lung cancer. Posttranscriptional regulations of HMGB1 gene are important for its function. After translation, HMGB1 undergoes some modifications, such as phosphorylation, ADP ribosylation, and acetylation. Acetylation of HMGB 1 potentially modulates HMGB1 to interact with other proteins both in nucleus and extracellularly (20).

In many cancers, HMGB1 is over-expressed depending on AGER, with which HMGB1 triggers a signal channel to boost the cancer-like oncoproteins (21). However, in our study, we found HMGB1 expression was overall downregulated in non-small cell lung cancer tissue not only at mRNA level but also at protein level. Interestingly, also making one exception, AGER is down-regulated in NSCLC. Why is HMGB1 gene expression different in NSCLC? The more perplexing question is how HMGB1 can act differently in cancers. HMGB1 has a paradoxical dual effect in cancer: on one hand it works like an oncoprotein to promote tumor progress and on the other hand it functions like an anticancer protein to trigger the protective anti-cancer T-cell responses. One explanation sounds reasonable: In most growing solid cancers, hypoxia kills cancer cells and dying cells release HMGB1 steadily, with which tumors are supported for proliferation, migration and neoangiogenesis. During chemotherapy and radiotherapy, large amounts of tumor cells die and massive HMGB1 release is rapid, so dendritic cells are recruited and activated, anti-neoplastic Tcell response is triggered. The kinetics of HMGB1 release (pulsed, when elicited by anti-neoplastic treatments and continuous, in progressively growing neoplasms) and the amount released in the unit of time (substantially higher when elicited by anti-neoplastic treatment) probably makes the difference (22). In lung cancer, apparently the AGER-HMGB1 signal channel is not dominant and some other pathways take its place to support cancers for proliferation, migration and neoangiogenesis.

During chemotherapy and radiotherapy, low HMGB1 expression indicates low release of HMGB1 protein, which may be one explanation of poorer therapeutical outcome in lung cancer than in other cancers. The evidence is that downregulation of HMGB 1 mRNA is especially stronger in patients with stage III-IV than those in stage I-II in our study. Analogously, Akaike et al found that in gastric cancer, the prognosis of the low HMGB-1 group $(n=41)$ was significantly poorer than that of the high HMGB-1 group $(\mathrm{n}=35, \mathrm{p}<0.05)$ (23). Our three patients who had a PR response in induction chemotherapy before the sections did not show particularly high expression of HMGB1. More patients should be included to survey the relationship between HMGB1 expression and outcome of chemotherapy or radiotherapy. Additional study should be conducted to find out whether low expression of 
HMGB1 can be used as an indicator for advanced progression or poor prognosis in lung cancer. While in human nasopharyngeal carcinoma, increased expression of HMGB1 is associated with advanced progression and poor prognosis (24). Several potential therapeutic methods targeting increased HMGB1 may come into clinical application soon, applying HMGB1-neutralizing antibodies (25), tuncated forms of HMGB1 (26), ethyl pyruvate (27) and sAGER (28) to block or replace HMGB1, inhibit HMGB1 release and block HMGB1-AGER signal channel. However, we suppose that it may be helpful to introduce exogenetic HMGB1 into the tumor mass during chemotherapy and radiotherapy especially in non-small cell lung cancer.

\section{Acknowledgements}

This study was supported by Natural Science Foundation of Jiangsu Province, China (BK2008326) and key project of medical science of Jiangsu Province, China (RC2007113). We thank Dr Decai Yu, Institute of Hepatobiliary Surgery, Nanjing University, for excellent technical assistance and advice.

\section{References}

1. Agresti A and Bianchi ME: HMGB proteins and gene expression. Curr Opin Genet Dev 13: 170-178, 2003.

2. Lotze MT and Tracey KJ: High-mobility group box 1 protein (HMGB1): nuclear weapon in the immune arsenal. Nat Rev Immunol 5: 331-342, 2005.

3. Klune JR, Dhupar R, Cardinal J, Billiar TR and Tsung A: HMGB1: endogenous danger signaling. Mol Med 14: 476-484, 2008.

4. Lotze MT, Zeh HJ, Rubartelli A, et al: The grateful dead: damage-associated molecular pattern molecules and reduction/ oxidation regulate immunity. Immunol Rev 220: 60-81, 2007.

5. Merenmies J, Pihlaskari R, Laitinen J, Wartiovaara J and Rauvala H: $30-\mathrm{kDa}$ heparin-binding protein of brain (amphoterin) involved in neurite outgrowth. Amino acid sequence and localization in the filopodia of the advancing plasma membrane. J Biol Chem 266: 16722-16729, 1991.

6. Taguchi A, Blood DC, del Toro G, et al: Blockade of AGERamphoterin signalling suppresses tumour growth and metastases. Nature 405: 354-360, 2000.

7. Riuzzi F, Sorci G and Donato R: The amphoterin (HMGB1)/ receptor for advanced glycation end products (AGER) pair modulates myoblast proliferation, apoptosis, adhesiveness, migration, and invasiveness. Functional inactivation of AGER in L6 myoblasts results in tumor formation in vivo. J Biol Chem 281: 8242-8253, 2006

8. Mitola S, Belleri M, Urbinati C, et al: Cutting edge: extracellular high mobility group box-1 protein is a proangiogenic cytokine. J Immunol 176: 12-15, 2006.

9. Flohr AM, Rogalla P, Meiboom M, et al: Variation of HMGB1 expression in breast cancer. Anticancer Res 21: 3881-3885, 2001.
10. Brezniceanu ML, Volp K, Bosser S, et al: HMGB1 inhibits cell death in yeast and mammalian cells and is abundantly expressed in human breast carcinoma. FASEB J 17: 1295-1297, 2003.

11. Kawahara N, Tanaka T, Yokomizo A, et al: Enhanced coexpression of thioredoxin and high mobility group protein 1 genes in human hepatocellular carcinoma and the possible association with decreased sensitivity to cisplatin. Cancer Res 56: 5330-5333, 1996.

12. Poser I and Bosserhoff $\mathrm{AK}$ : Transcription factors involved in development and progression of malignant melanoma. Histol Histopathol 19: 173-188, 2004.

13. Poser I, Golob M, Buettner R and Bosserhoff AK: Upregulation of HMG1 leads to melanoma inhibitory activity expression in malignant melanoma cells and contributes to their malignancy phenotype. Mol Cell Biol 23: 2991-2998, 2003.

14. Choi YR, Kim H, Kang HJ, et al: Overexpression of high mobility group box 1 in gastrointestinal stromal tumors with KIT mutation. Cancer Res 63: 2188-2193, 2003.

15. Meyer A, Staratschek-Jox A, Springwald A, et al: Non-Hodgkin lymphoma expressing high levels of the danger-signalling protein HMGB1. Leuk Lymphoma 49: 1184-1189, 2008.

16. Volp K, Brezniceanu ML, Bosser S, et al: Increased expression of high mobility group box 1 (HMGB1) is associated with an elevated level of the antiapoptotic c-IAP2 protein in human colon carcinomas. Gut 55: 234-242, 2006.

17. Apetoh L, Ghiringhelli F, Tesniere A, et al: Toll-like receptor 4-dependent contribution of the immune system to anticancer chemotherapy and radiotherapy. Nat Med 13: 1050-1059, 2007.

18. Bartling B, Hofmann HS, Weigle B, Silber RE and Simm A: Down-regulation of the receptor for advanced glycation endproducts (AGER) supports non-small cell lung carcinoma. Carcinogenesis 26: 293-301, 2005.

19. Muller S, Ronfani L and Bianchi ME: Regulated expression and subcellular localization of HMGB1, a chromatin protein with a cytokine function. J Intern Med 255: 332-343, 2004.

20. Ulloa L and Messmer D: High-mobility group box 1 (HMGB1) protein: friend and foe. Cytokine Growth Factor Rev 17: 189-201, 2006.

21. Ellerman JE, Brown CK, de Vera M, et al: Masquerader: high mobility group box-1 and cancer. Clin Cancer Res 13: 2836-2848, 2007.

22. Campana L, Bosurgi L and Rovere-Querini P: HMGB1: a twoheaded signal regulating tumor progression and immunity. Curr Opin Immunol 20: 518-523, 2008.

23. Akaike H, Kono K, Sugai H, et al: Expression of high mobility group box chromosomal protein-1 (HMGB-1) in gastric cancer. Anticancer Res 27: 449-457, 2007.

24. Wu D, Ding Y, Wang S, Zhang Q and Liu L: Increased expression of high mobility group box 1 (HMGB1) is associated with progression and poor prognosis in human nasopharyngeal carcinoma. J Pathol 216: 167-175, 2008.

25. Wang H, Bloom O, Zhang M, et al: HMG-1 as a late mediator of endotoxin lethality in mice. Science 285: 248-251, 1999.

26. Yang H, Ochani M, Li J, et al: Reversing established sepsis with antagonists of endogenous high-mobility group box 1 . Proc Natl Acad Sci USA 101: 296-301, 2004.

27. Ulloa L, Ochani M, Yang H, et al: Ethyl pyruvate prevents lethality in mice with established lethal sepsis and systemic inflammation. Proc Natl Acad Sci USA 99: 12351-12356, 2002.

28. Hanford LE, Enghild JJ, Valnickova Z, et al: Purification and characterization of mouse soluble receptor for advanced glycation end products (sAGER). J Biol Chem 279: 50019-50024, 2004. 